\title{
Un enfoque lúdico de la educación científica y matemática globalizada en infantil: La coci-ciencia
}

\author{
Virginia Molina Correa, Alicia Fernández Oliveras y María Luisa Oliveras. Universidad de \\ Granada \\ 口 \\ Recepción: 12 de abril de 2015 | Revisión: 30 de mayo de 2015 | Aceptación/Publicación: 24 de julio de 2015 \\ Correspondencia: virgimc@correo.ugr.es | alilia@ugr.es | oliveras@ugr.es | http://hdl.handle.net/10481/37118
}

\begin{abstract}
Resumen: Hemos planteado un recurso lúdico con el fin de integrar la enseñanza de las ciencias y las matemáticas en la etapa de Educación Infantil. El juego está contextualizado en uno de los lugares más presentes en la vida diaria de los niños: la cocina. Apoyamos nuestra propuesta en las ideas de autores como Rousseau Montessori, Froebel y, especialmente, Decroly que introdujo los "centros de interés" y los materiales de uso común en la vida cotidiana como recursos didácticos. Las principales características del juego diseñado se muestran en dos fichas de archivo como las que podrían emplearse en la gestión de los recursos disponibles en una ludoteca.
\end{abstract}

Palabras clave: Didáctica de las Ciencias Experimentales | Didáctica de la Matemática | Formación del Profesorado | Juegos Educativos

A PLAYFUL APPROACH TO INTEGRATED SCIENCE AND MATHEMATICS EDUCATION IN KINDERGARTEN: THE COCI-CIENCIA

Abstract: We have proposed a playful resource in order to integrate the teaching of science and mathematics in kindergarten. The game is contextualized in one of the most important places in the daily life of children: the kitchen. We are basing our proposal on the ideas of authors like Rousseau, Montessori, Froebel and, especially, Decroly, who introduced the "centres of interest" and the materials commonly used in everyday life as teaching resources. The main features of the game designed are shown on two index cards such as those that might be used in the management of the resources available in a playgroup centre.

Keywords: Science Education | Mathematical Education | Teacher Training | Educational Games

Agradecimientos: Al Secretariado de Innovación Docente del Vicerrectorado de Ordenación Académica y Profesorado de la Universidad de Granada (España), por conceder el Proyecto de Innovación Docente 2013-2016.

\section{Introducción}

En la etapa de Educación Infantil, el niño se va desarrollando de forma autónoma, creando una estructura de su yo, para adaptarse al mundo que le rodea y poder vivir en armonía con él mismo y su entorno. Según la psicología cognitiva, el juego constituye una fuente de conocimiento muy importante sobre todo en los períodos sensorio-motriz y pre-operacional. También, cumple una función biológica importante: el ejercicio de todos los órganos y capacidades. Cuando el niño manipula un objeto y juega con él, está interpretando un argumento. Cuando juegan juntos varios niños, están asimilando el lenguaje, la comunicación, y aprenden a coordinar sus acciones entre todos.

Las áreas de Educación Infantil: el conocimiento de sí mismo y autonomía personal, el conocimiento de su entorno y la comunicación y representación mediante el lenguaje, como bien se indica en el Real Decreto 1630/2006 (2007), "deben trabajarse de manera conjunta e integrada" (p. 480) y "promover la imagen positiva de uno mismo, la autonomía, la conciencia de la propia competencia" (p. 476). Para que ello sea posible, consideramos crucial la integración de las ciencias y las matemáticas en las propuestas de enseñanza de Educación Infantil y nos parece idóneo hacerlo con una metodología basada en el juego, tal y como apuntan las concepciones de maestros en formación (Fernández-Oliveras y Oliveras, 2014b, 2015).

En este artículo resumimos el diseño y la elaboración de un recurso lúdico para la enseñanza globalizada de las ciencias y las matemáticas en Educación Infantil, 
contextualizado en uno de los lugares más presentes en la vida diaria de los niños: la cocina. Apoyamos nuestra propuesta en las ideas de Rousseau (1985), ya que están aprendiendo matemáticas y ciencias mediante la experimentación. Además, este defendía la autonomía. También en María Montessori (1937), que defendía el desarrollo de los sentidos y aprender mediante ensayo-error, y en Decroly (1965), que introdujo los centros de interés y los materiales de uso común en la vida cotidiana. Así como en las tesis de Froebel (1902) ya que, a la vez que el niño está en su propia naturaleza, aprende a través el juego. Las características esenciales del recurso diseñado se muestran en dos fichas cuyos modelos proceden de las actividades de una asignatura de $4^{\circ}$ curso del Grado en Maestro de Educación Infantil de la Universidad de Granada (Fernández-Oliveras y Oliveras, 2014a)

\section{DISEÑO Y ELABORACIÓN DEL JUEGO}

Una vez propuesta la creación de un juego educativo que tratara contenidos matemáticos-científicos se sopesó en qué ámbito de la vida del niño se podían experimentar dichos contenidos con más frecuencia.

Tras un tiempo de reflexión, surgió la idea de usar experimentos científicos llegando a la conclusión de escoger seis experimentos o pruebas seguidos de un premio final por haberlos completado: fabricar "masa de sal". Se decidió relacionar estos experimentos con cada uno de los ingredientes necesarios para hacer la "masa de sal" y organizarlos por rincones.

El juego fue construido de manera artesanal. Una vez escogidos los experimentos que se iban a hacer, lo primero que se elaboró fue el tablero de familiarización. A continuación se empezaron a preparar los instrumentos necesarios para las actividades planteadas. En primer lugar, se pintaron los cubiertos de plástico necesarios para la seriación. Más tarde, se prepararon dos botellas de plástico de agua vacías poniéndoles un globo a cada una en la boquilla y abriendo un agujero en la parte de abajo de una de ellas. Además, se prepararon otras dos botellas más para la prueba de agua salada y agua dulce.

Cuando ya teníamos esto finalizado y todo lo pintado seco, se dibujó en cartulina una nevera, varios alimentos y un vaso, con los correspondientes ingredientes utilizados en la disolución. Más tarde se colorearon y recortaron estos. Así mismo, se dibujó una medalla en un folio, del que se hicieron 25 copias (correspondiendo al número total de alumnos).

Así, una vez terminado todo lo manual, nos dirigimos a buscar todos los demás materiales y a meterlos en bolsas para llevarlos al aula: aceite, sal, espaguetis, bolsa de ensalada, harina, vasos de plástico, barreño, patatas, pajitas, cuchillo, colorante, alcohol, macarrones, y varios más. Llevo una semana preparar todos los materiales, en ratos libres, siendo fácil la creación del recurso completo.

\section{FICHAS TÉCNICA Y DIDÁCTICA DEL JUEGO}

Las características esenciales del juego diseñado se muestran en las Tablas 1 y 2. Dichas tablas constituyen fichas de archivo como las que podrían emplearse en la gestión de los recursos disponibles en una ludoteca. 
Tabla 1. Ficha técnica del juego "La coci-ciencia".

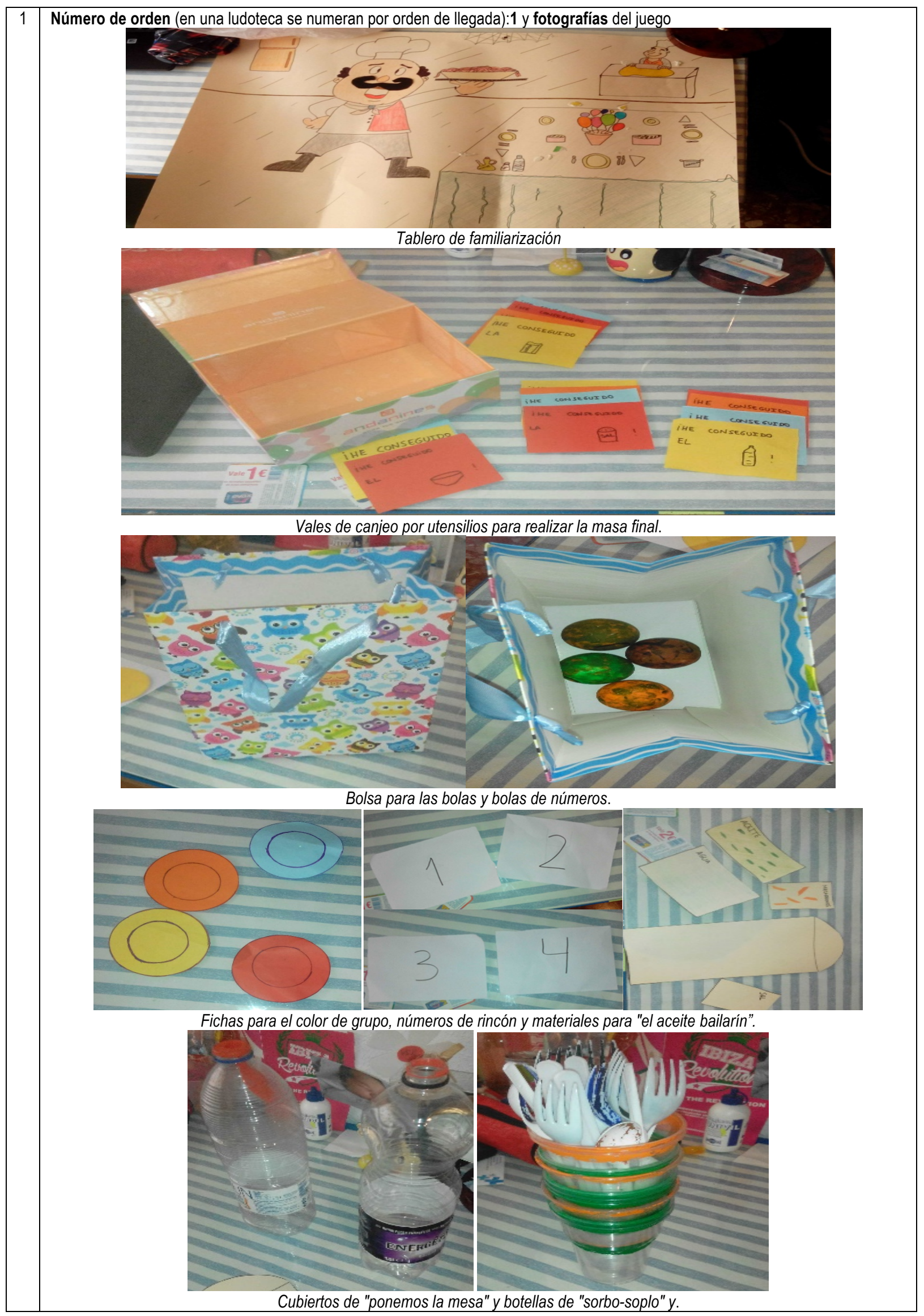




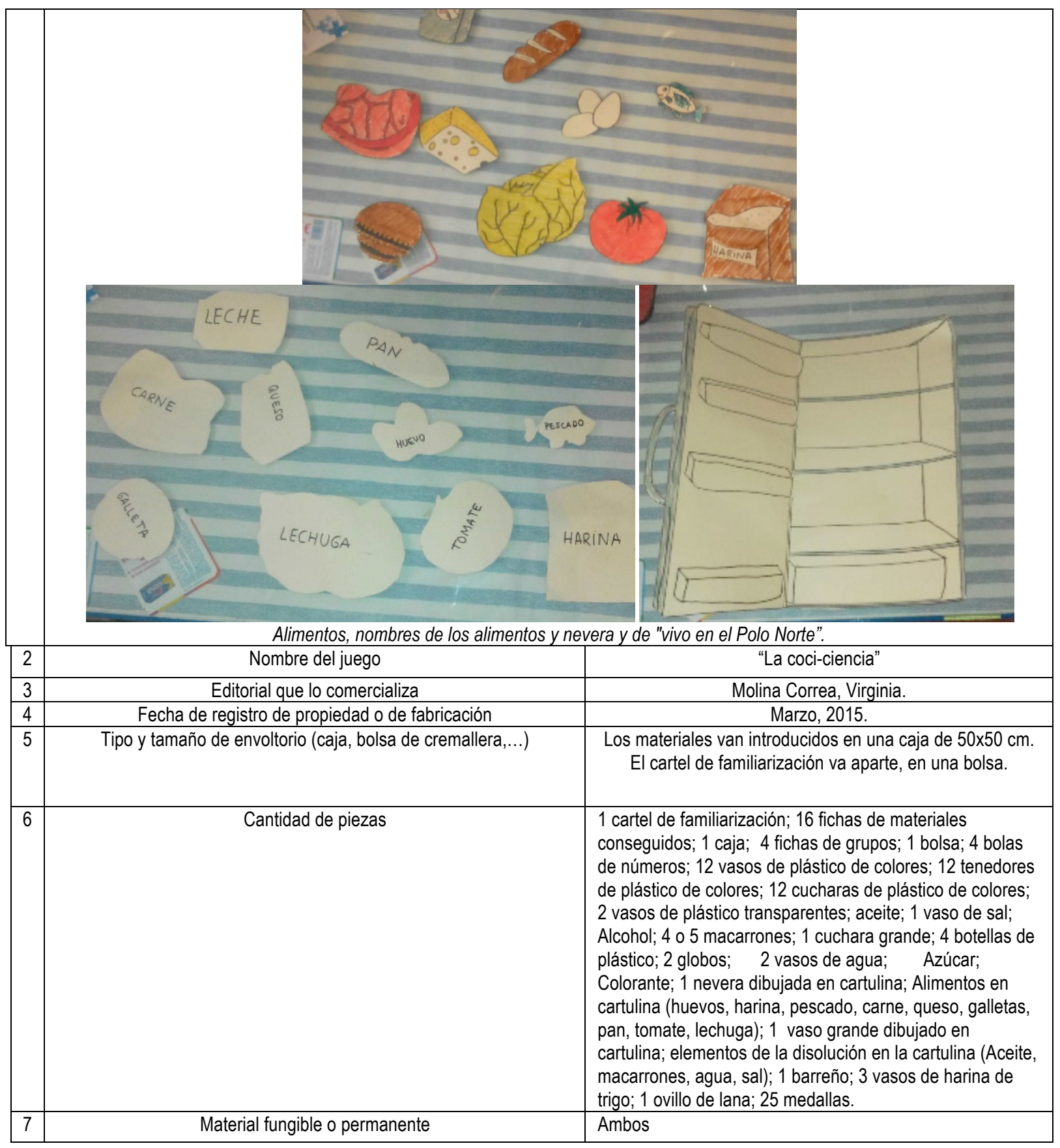

Tabla 2. Ficha didáctica del juego "La coci-ciencia".

\begin{tabular}{|l|l|}
\hline Nombre del juego & \multicolumn{1}{|c|}{ "La coci-ciencia" } \\
\hline Piezas y material: descripción & $\begin{array}{l}\text { Este juego contiene las siguientes piezas: 1 cartel; 16 fichas que indican los materiales } \\
\text { conseguidos; } 1 \text { caja; } 4 \text { fichas para indicar el color de grupo; } 1 \text { bolsa para meter las } 4 \text { bolas } \\
\text { indicando el número de rincón; } 12 \text { vasos de plástico de colores; } 12 \text { tenedores de plástico de } \\
\text { colores; } 12 \text { cucharas de plástico de colores; } 2 \text { vasos de plástico transparentes; aceite; } 1 \text { vaso de } \\
\text { sal; alcohol; } 4 \text { o } 5 \text { macarrones; } 1 \text { cuchara grande; } 4 \text { botellas de plástico; } 2 \text { globos; } \\
2 \text { vasos de agua; azúcar; colorante; } 1 \text { nevera dibujada en cartulina; alimentos en cartulina } \\
\text { (huevos, harina, pescado, carne, queso, galletas, pan, tomate, lechuga); } 1 \text { vaso grande } \\
\text { dibujado en cartulina; elementos de la disolución en la cartulina (Aceite, macarrones, agua, sal); } \\
1 \text { barreño; } 3 \text { vasos de harina de trigo; } 1 \text { ovillo de lana; y 25 medallas dibujadas en un folio. }\end{array}$ \\
\hline $\begin{array}{l}\text { Lugar de fabricación } \\
\text { pamologación [Sí, en qué }\end{array}$ & España \\
\hline
\end{tabular}




\begin{tabular}{|c|c|}
\hline $\begin{array}{l}\text { Cualidades intrínsecas de las } \\
\text { piezas: formas y color, } \\
\text { material del que están hechas }\end{array}$ & $\begin{array}{l}\text { Las materiales de los que están hechos las piezas del juego son los siguientes: } \\
\text { Plástico: Vasos, cucharas, tenedores, bolas de números, bolsa, barreño, botellas y globos. } \\
\text { Cartón/cartulina: caja, tablero principal, nevera, alimentos, vaso, ingredientes disolución, } \\
\text { Papel: fichas de los grupos y medallas. } \\
\text { Otros: Sal, aceite, harina, agua, azúcar, colorante, lana, alcohol y macarrones. } \\
\text { La mayoría de las piezas son de plástico y de cartón/cartulina, materiales apropiados para los } \\
\text { niños, ya que son blandos. Además, no existen piezas pequeñas las cuales puedan ser } \\
\text { ingeridas, sino que todas tienen un tamaño adecuado. Las formas y colores son diversos }\end{array}$ \\
\hline $\begin{array}{l}\text { Cualidades relativas de las } \\
\text { piezas: tamaños, texturas, } \\
\text { volumen }\end{array}$ & $\begin{array}{l}\text { El tamaño de las piezas es adecuado a la edad, no siendo estas demasiado pequeñas ni } \\
\text { demasiado grandes. } \\
\text { La textura es muy variada, tanto suave, como rugoso. Además también hay piezas blandas (la } \\
\text { masa, harina, entre otras) y piezas duras (cubiertos). }\end{array}$ \\
\hline $\begin{array}{l}\text { Dinámica del juego y reglas: } \\
\text { explicación }\end{array}$ & $\begin{array}{ll}\text { Reglas: } \\
\text { - } & \text { Cuidar y respetar el material. } \\
\text { - } & \text { Usar el material de forma adecuada. } \\
\text { - } & \text { Seguir los pasos que guie la maestra. } \\
\text { - } & \text { No ingerir ningún producto. } \\
\text { - } & \text { Mantenerse en el grupo. } \\
\text { - } & \text { Seguir el orden propuesto. } \\
\text { - } & \text { Respetar a los compañeros. } \\
\text { El juego se articula mediante diferentes rincones en los que se realizarán experimentos con } \\
\text { elementos y utensilios de cocina, como pruebas a superar para obtener los ingredientes y } \\
\text { materiales para el experimento final. Los rincones son: "ponemos la mesa" (realización de } \\
\text { seriaciones con cubiertos), "el aceite bailarín" (comprobación del comportamiento de fluidos } \\
\text { según su densidad), "sorbo-soplo" (experimentación con el aire contenido en una botella), "vivo } \\
\text { en el Polo Norte" (clasificación de alimentos y refrigeración) y "somos auténticos cocineros" } \\
\text { (elaboración de masa de sal). }\end{array}$ \\
\hline $\begin{array}{l}\text { Peligrosidad/riesgos (explicar } \\
\text { si en las piezas o en su uso } \\
\text { con determinadas reglas) } \\
\end{array}$ & $\begin{array}{l}\text { Algún niño puede ingerir la disolución. } \\
\text { Pueden darle un mal uso a los tenedores de plástico, e hincárselos a algún compañero, o ellos } \\
\text { mismos. }\end{array}$ \\
\hline Tiempo de preparación & 5 minutos \\
\hline Duración [corta/media/larga] & Larga. \\
\hline $\mathrm{N}^{0}$ de jugadores & Los que se quiera. \\
\hline $\begin{array}{l}\text { Edad preferente de los } \\
\text { jugadores }\end{array}$ & 4-6 años \\
\hline De uso en interior/exterior & Interior. \\
\hline $\begin{array}{l}\text { Tipos de acciones de los } \\
\text { jugadores primordialmente } \\
\text { (físicas, mentales, ambas) }\end{array}$ & Ambas. \\
\hline Sentidos que se utilizan & Tacto, gusto, vista, oído. \\
\hline $\begin{array}{l}\text { Habilidades/capacidades } 0 \\
\text { destrezas que favorece } \\
\text { (psicomotrices, cognitivas...) } \\
\end{array}$ & $\begin{array}{l}\text { Favorece la Psicomotricidad fina, psicomotricidad gruesa, habilidad óculo-manual y viso-motora, } \\
\text { lógica, Concentración, atención, creatividad, interacción social, cognición, }\end{array}$ \\
\hline Objetivos propios del juego & $\begin{array}{l}\text { Experimentar con el concepto de mezcla. Trabajar la seriación. Establecer correspondencias. } \\
\text { Adquirir conocimientos sobre cambios en la materia. Experimentar con los conceptos de } \\
\text { densidad, flotabilidad y disolución. Experimentar con el volumen que ocupa el aire, y con los } \\
\text { conceptos de presión y elasticidad. Familiarizarse con los conceptos de refrigeración y } \\
\text { descomposición de la materia orgánica. Desarrollar el sentido del gusto. Desarrollar de la } \\
\text { creatividad. Desarrollar la autonomía. Desarrollar la atención. Trabajar en grupo. Desarrollar de } \\
\text { la psicomotricidad gruesa y fina. Desarrollar la capacidad de expresión. }\end{array}$ \\
\hline $\begin{array}{l}\text { Coste económico (estimado o } \\
\text { conocido) }\end{array}$ & $25 €$ \\
\hline Contenidos matemáticos & $\begin{array}{l}\text { Pensamiento lógico-matemático: seriación, ordenación, correspondencias. Pensamiento } \\
\text { espacio-temporal y causal: la medida, estimación de medidas, el todo y las partes. Pensamiento } \\
\text { numérico: estimación de cantidades, conteo. }\end{array}$ \\
\hline Contenidos científicos & $\begin{array}{l}\text { La materia: estados, propiedades y cambios, volumen, color, olor, sabor, color, dureza, } \\
\text { fragilidad, elasticidad. Presión. Medidas de longitud y volumen. Mezclas de colores. Los } \\
\text { sentidos. Salud y ecología. }\end{array}$ \\
\hline $\begin{array}{l}\text { Autocorrección [Sí, de qué } \\
\text { forma:... / No] }\end{array}$ & $\begin{array}{l}\text { Si, ya que para superar las pruebas tienen que completar una serie de rincones, y entre todos } \\
\text { se van corrigiendo unos a otros, y con ellos mismos. }\end{array}$ \\
\hline $\begin{array}{l}\text { Universalidad en sus reglas o } \\
\text { composición: puede ser } \\
\text { comprendido sin } \\
\text { explicaciones [Sí/No] }\end{array}$ & No \\
\hline $\begin{array}{l}\text { Fundamentación teórica } \\
\text { (autores en los que se basa) }\end{array}$ & i (1937), Decroly (1965 \\
\hline
\end{tabular}




\section{Reflexión final}

En este artículo se ha mostrado de forma resumida el planteamiento de un recurso lúdico para abordar, con un enfoque integrador, el tratamiento de las matemáticas y las ciencias en Educación Infantil.

\section{Referencias}

Decroly O. (1965). La Fonction de Globalisation et l'Enseignement. Brussels: Editions Desoer.

Fernández-Oliveras, A. y Oliveras, M. L. (2014a). Playing for science and mathematics education: an experience for pre-service kindergarten teacher training. En Costa M. F. M., Pombo P., Dorrío B.V. (Eds.), Hands-on Science. Science Education with and for Society (180 -183). Braga: Hands-on Science Network.

Fernández-Oliveras, A. y Oliveras, M. L. (2014b). Pre-service kindergarten teachers' conceptions of play, science, mathematics, and education. Procedia - Social and Behavioral Sciences, 152, 856-861.

Fernández-Oliveras, A. y Oliveras, M. L. (2015). Conceptions of science, mathematics, and education of prospective kindergarten teachers in a play-based training. International Journal on Advances in Education Research, 2(1), 37-48.

Froebel, F. (1902). La educación del hombre. Nueva York: D. Appleton y Compañía.

Montessori, M. (1937). El método de la pedagogía científica aplicado a la educación. Barcelona: Araluce.

Real Decreto por el que se establecen las enseñanzas mínimas del segundo ciclo de Educación infantil. (Real Decreto 1630/2006, 29 de diciembre). Boletín Oficial del Estado, n4, 2007, 4 enero.

Rousseau, J. J. (1985). Emilio o de la Educación. Madrid: Edaf. 